Portland State University

PDXScholar

\title{
Voice Measures and Listener Acceptance of Tracheoesophageal Speech
}

Heidii llona Roberts

Portland State University

Follow this and additional works at: https://pdxscholar.library.pdx.edu/open_access_etds

Part of the Communication Sciences and Disorders Commons

Let us know how access to this document benefits you.

\section{Recommended Citation}

Roberts, Heidii Ilona, "Voice Measures and Listener Acceptance of Tracheoesophageal Speech" (1997). Dissertations and Theses. Paper 5827.

https://doi.org/10.15760/etd.7697

This Thesis is brought to you for free and open access. It has been accepted for inclusion in Dissertations and Theses by an authorized administrator of PDXScholar. Please contact us if we can make this document more accessible: pdxscholar@pdx.edu. 


\section{THESIS APPROVAL}

The abstract and thesis of Heidii Ilona Roberts for the Master of Science in Speech Communication: Speech and Hearing Science were presented February 6, 1997, and accepted by the thesis committee and the department.

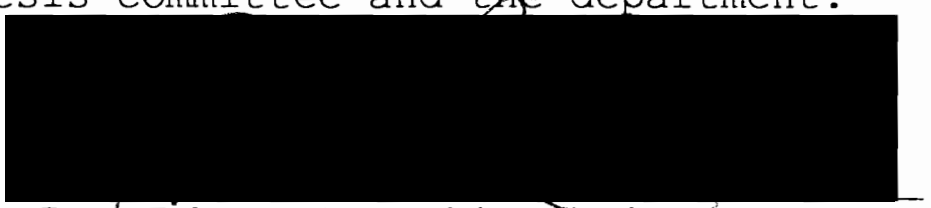

Dr. John Tetnowski, Chair

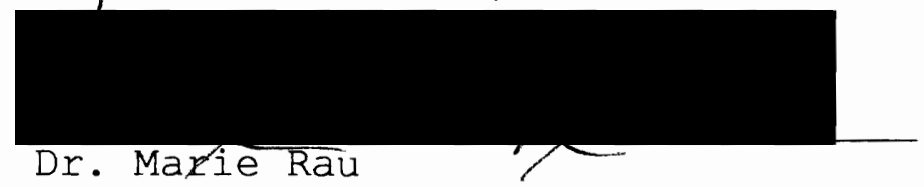

Dr. Marie Rau

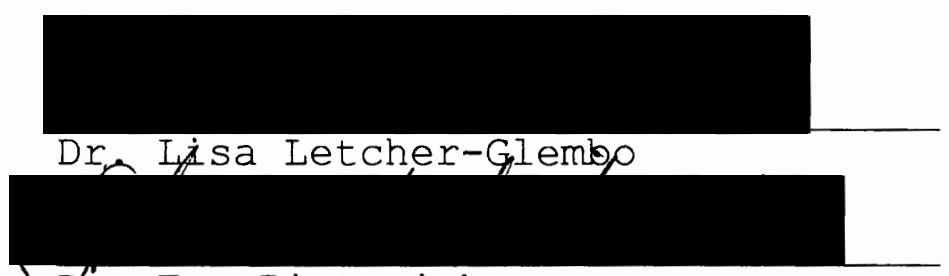

Dr. Tom Dieterich

Representative of the Office of Graduate Studies

APPROVED:

Dr. Stepheth A. Kos8koff, Chair UT Department of Speech Communication

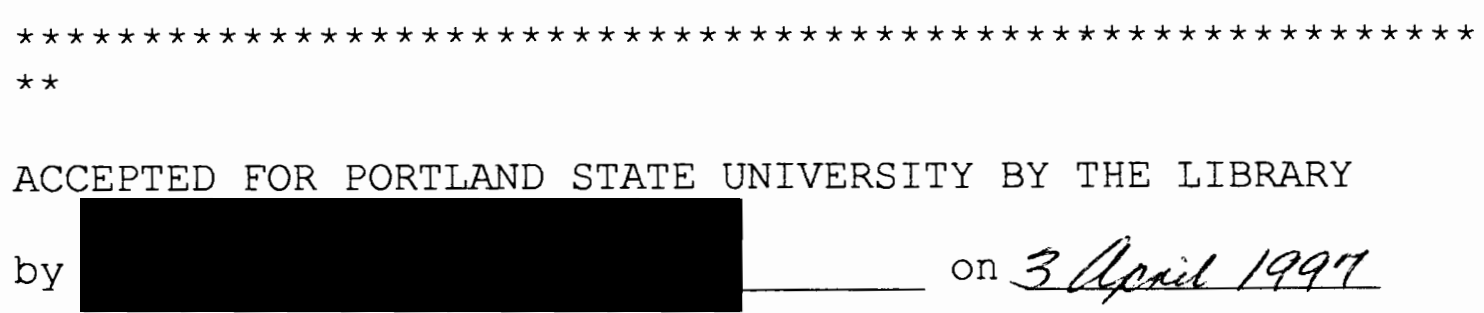




\section{ABSTRACT}

An abstract of the thesis of Heidii Ilona Roberts for the Master of Science in Speech Communication: Speech and Hearing Science presented February 6, 1997.

Title: Voice Measures and Listener Acceptance of Tracheoesophageal speech

The purpose of this study was to investigate the relationship of perturbation measures, jitter and shimmer, and ratings of acceptability by naive listeners. Six tracheoesophageal (TEP) speakers and 14 naive listeners participated in the study. Each speaker was recorded while reading The Rainbow passage. The six samples of voice were then presented to the listeners for rating. The naive listeners used an equal appearing seven pint scale to rate voice acceptability.

No significant correlation was found between the perturbation measures and the ratings of acceptability. Due to the small subject size in this study, the findings 
are inconclusive. The results due suggest that methods of predicting and judging voice quality in TEP speakers are not the same as those used for judging voice quality in laryngeal speakers. 


\title{
VOICE MEASURES AND LISTENER ACCEPTANCE OF
}

TRACHEOESOPHAGEAL SPEECH

\author{
by \\ HEIDII ILONA ROBERTS
}

A thesis submitted in partial fulfillment of the requirements for the degree of

MASTER OF SCIENCE

in

SPEECH COMMUNICATION:

SPEECH AND HEARING SCIENCE

Portland State University

1997 


\section{ACKNOWLEDGMENTS}

I would like to thank Dr. Tetnowski for sharing his knowledge of research and for his confidence in completing this project. His understanding of the research and his knowledge of instrumentation made this study interesting and motivating.

I would also like to extend a special thank-you to Dr. Rau and Donna Graville for taking time out of their busy schedules to answer questions and help locate subjects for participation. Their assistance is very much appreciated. My appreciation also goes to Dr. LetcherGlembo and Dr. Dieterich for their flexibility in schedules and their assistance in reviewing this research.

I would like to acknowledge and thank all the subjects and judges who took part in this study. Without their interest and willingness to dedicate personal time this thesis would not be possible. My dear friend Jennifer Peterson also requires special acknowledgment for her constant support, humor, and training on the computer analysis program. 
My family, co-workers, and friends deserve my gratitude for their support, understanding, patience, and encouragement. They have made this journey easier.

My last and dearest thank-you goes to my husband and best friend, Mike, who provided comfort, support, and a helpful hand during the evolution of this project. 
TABLE OF CONTENTS

ITEM

$\underline{\text { PAGE }}$

ACKNOWLEDGMENTS ..................... ii

LIST OF TABLES.......................

CHAPTER I: INTRODUCTION $\ldots \ldots \ldots \ldots \ldots \ldots \ldots \ldots$

Statement of Purpose...............4

Definition of Terms................ 4

CHAPTER II: REVIEW OF THE LITERATURE.......... 8

Aerodynamic and Myoelastic Characteristics.. 8

Trans-source Airflow Rates......... 8

Airway Resistance............... 10

Acoustic Measures................... 12

Fundamental Frequency............ 13

Jitter Measurements.............. 15

Shimmer Measurements............. 17

Perceptual Judgments................ 20

CHAPTER III: METHODS AND PROCEDURES......... 24

General Plan.................... 24

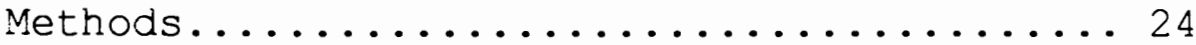

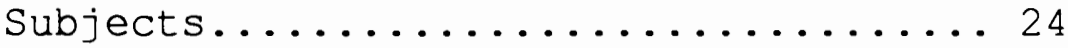

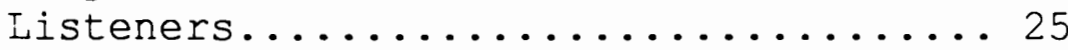

Instrumentation.............. 26

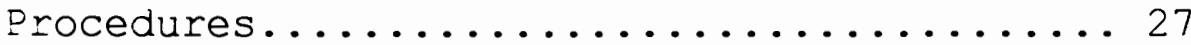

Recording Subjects............. 27

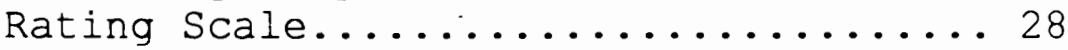

Interjudge Reliability........... 29

CHAPTER IV: RESULTS AND DISCUSSION......... 32

Results...................... 32

Discussion.................... 34

CHAPTER V: SUMMARY AND IMPLICATIONS......... 38

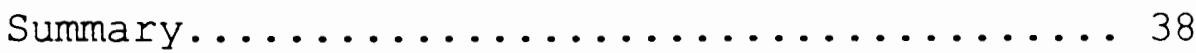

Implications................... 40

Clinical................... 40

Research...................4 41

REFERENCES.................... 43 APPENDICIES

A ACOUSTIC MEASURES .............. 46

B ACOUSTIC DATA................. 47

C TRACHEOESOPHAGEAL SUBJECT QUESTIONNAIRE.. 48 
D IISTENER SCREENING OUESTIONNAIRE......4 9

E ListeneR RATING SCALE............. 50 


\section{LIST OE TABLES}

TABLE

$\underline{P A G E}$

I Biographical Information of Speakers....... 25

II Biographical Information of Judges......... 26

II Interjudge Reliability Perturbation Measures. 30

IV Jitter Mean, Standard Deviation, and Range... 33

V Shimmer Mean, Standard Deviation, Range...... 34

VI Acoustic Measures and Naive Listener Judgements of Acceptability ............. 34 


\section{CHAPTER I}

\section{INTRODUCTION}

Total or partial removal of the larynx may be indicated when cancer is present in the larynx or laryngeal area. A total laryngectomy involves removal of the entire cartilaginous larynx, its intrinsic muscles and membranes, the hyoid bone, and also may involve two or three rings of the trachea (Prater \& Swift, 1984). When the cancer has spread to tissue and glands surrounding the larynx, surgical removal of additional muscles, nerves, and glands may be indicated. The top of the remaining trachea is sewn to the base of the neck to create a permanent opening (tracheostoma). Respiration then occurs through the stoma instead of through the nose and mouth. The laryngectomee must make many adjustments in physiological and psychosocial aspects of speech and everyday living. Several methods of voice restoration are available such as an artificial larynx, esophageal speech, and tracheoesophageal (TEP) speech.

The first reported use of the artificial larynx was in 1859 (Keith \& Shanks, 1986). The patient articulated while pulmonary 
air was exhaled from the tracheostoma into a tube containing a reed, thus producing a tone which could be shaped by the articulators to produce oral speech (Lerman, 1991). Today artificial larynxes are categorized as intraoral (tube in mouth during articulation), transcervical (electrolarynx creates vibration against the neck during articulation), or pneumatic artificial larynx (pulmonary air travels up through the tracheostoma and into the voice prosthesis which is placed over the stoma) (Lerman, 1991).

In the $1930^{\prime}$ 's, esophageal speech became an alternative to the electrolarynx. During esophageal speech, air is taken into the upper esophagus and expelled past the pharyngoesophageal segment or junction (neoglottis) causing it to vibrate. The articulated vibrations create oral speech for the laryngectomized individual. The limitation set by the size of the esophagus restricts the amount of air available for articulation.

The problem of air reservoir limitation was partially resolved during the early $1980^{\prime} \mathrm{s}$ when the tracheoesophageal puncture (TEP) was introduced as a method of speech communication. A one-way valve is 
inserted into a surgically created passage way between the trachea and esophagus so that air from the lungs may be used for speech. Traditional esophageal and tracheoesophageal (TEP) speech both use the same voicing segment but they differ aerodynamically.

Tracheoesophageal speech has the aerodynamic advantages of pulmonary air support, a closed airway, and the use of the chest wall during speech production. These advantages allow the TEP speaker

to produce speech that more closely resembles laryngeal speech aerodynamically as well as acoustically (Doyle, Danhhuer, \& Reed 1988; Robbins, Fisher, Blom, \& Singer 1984; Bags \& Pine, 1983). This does not mean, however, that TEP speech is more perceptually acceptable than esophageal speech (Trudeau, 1987).

Researchers have traditionally used quantifiable variables such as fundamental frequency, vocal intensity, and/or duration to describe the characteristics of alaryngeal speech and to distinguish differences between esophageal, tracheoesophageal, and laryngeal speech (Robbins et al., 1984a). However, little research has been published describing the acoustic characteristics of TEP speech or the effects of these measures on listener 
perceplion. Further research is needed to expand our knowledge of the relationship between acoustic characteristics and the effect those characteristics have on naive listener perceptions of voice quality.

\section{STATEMENT OF PURPOSE}

The purpose of this research is to determine if there is a relationship between listener judgments of acceptability and acoustic measures found in tracheoesophageal speech. The following questions were asked:

1. Is there a significant correlation between naive listener ratings of tracheoesophageal speech acceptability and jitter percent of tracheoesophageal speech?

2. Is there a significant correlation between naive listener ratings of tracheoesophageal speech acceptability and shimmer percent of tracheoesophageal speech?

\section{DEFINITIONS}

The following definitions are presented to help clarify terms found in this study: 
Amplitude Perturbation (Vocal Shimmer): variations in cycle-to-cycle amplitude difference measured during sustained phonation (Orlikoff \& Baken, 1993). It can be calculated using the amplitude perturbation measure:

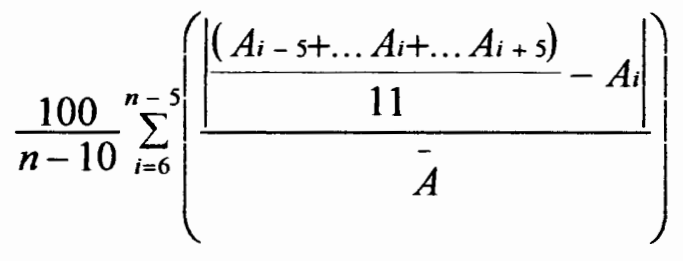

$$
\bar{A}=\left(\frac{1}{n}\right) \sum_{i=1}^{n} A_{i}
$$

$$
\mathrm{A}_{\mathrm{i}}=\text { Peak-Peakamplitudeithcycle }
$$

Deglutition: The act of swallowing.

Esophageal speech: Voice produced by injecting or inhaling air into the esophagus and articulating upon its release. Frequency Perturbation (Vocal Jitter): The variability in the fundamental frequency that occurs from one cycle to the next during vocal production.

It is partially responsible for the listeners' perception of harsh, hoarse, or rough vocal quality (Orlikoff \& Baken, 1993).

This variability during vocal production can be calculated using the frequency perturbation measure: 


$$
\frac{\frac{1}{n-2}\left[\sum_{i=2}^{n-1}\left|t_{i-1+t_{i}+t_{i+1}}^{3}-t_{i}\right|\right]}{\frac{1}{n} \sum_{i=1}^{n} t_{i}} X 100
$$

Hypopharynx: The lower area of the pharynx.

Laryngectomee: A person who has had their larynx removed. Laryngectomy: The surgical removal of the larynx.

Larynx: a muscular and cartilaginous structure at the upper area of the trachea which houses the vocal folds. It is also commonly known as the "voice box".

Neoglottis: The new voice source after a laryngectomy. Oscillograph: A printed representation of the vocal signal as a waveform.

Pharynx: The membranous tube that connects the mouth and nares with the esophagus.

Perturbation: Measures that serve to quantify short-term instability of the vocal signal (Baken, 1987). These measurements are used to describe hoarseness of voice and other vocal abnormalities.

Quasi-periodic: a sound wave that has a cycle to cycle presentation.

Relative Average Perturbation (\% Jitter): A relative jitter measure that additionally attempts to smooth the 
long-term fundamental frequency changes. The long-term effects may be associated with the relatively regular upward and downward variations as associated with a tremor (Orlikoff \& Baken, 1993).

This measurement is calculated:

$$
\frac{100}{n-2} \sum_{i=2}^{n-1}\left(\frac{\left.\left|\frac{\left(P_{i}-1+P_{i}+P_{i+1}\right)}{3}-P_{i}\right|\right)}{\bar{P}}\right) \quad \bar{P}=\left(\frac{1}{n}\right) \sum_{i=1}^{n} P_{i}
$$

Trachea: A tube-like cartilagenous structure which extends from the larynx to the bronchi. It serves as the principal passageway for air to and from the lungs. Tracheoesophageal speech (TEP): Speech produced by directing pulmonary air through a one-way valve to the esophagus for speech.

Trans-source airflow: The flow of air as it travels through the trachea and esophagus to exit the mouth. 
CHAPTER II

REVIEW OF THE LITERATURE

The following literature review is presented to provide a basic understanding of tracheoesophageal (TEP) speech and esophageal speech. This chapter will present findings that suggest TEP speech is more acoustically similar to laryngeal speech than is esophageal speech. It will investigate how people perceive TEP speech compared to esophageal speech and which is judged more acceptable. Little research is available that examines this acceptance along with certain acoustic measures of alaryngeal speech, such as jitter and shimmer.

AERODYNAMIC AND MYOELASTIC CHARACTERISTICS

\section{Trans-source Airflow Rates}

Tracheoesophageal speakers are able to use their pulmonary air for speech. The advantages of this capability include increased respiratory support to power speech 1400 $1000 \mathrm{cc}$ of air in the lungs), a closed airway, and the advantageous chest wall and background forces of the human respiratory system (Weinberg, Horii, Blom, \& Singer, 1982). 
[Blank] 
Traditional esophageal speakers are therefore at a disadvantage because the average size and elasticity of the esophagus can hold approximately $80 \mathrm{cc}$ of air (Van den Berg \& Moolenaar, 1959). Additionally the person's ability to control the neoglottis while releasing air for esophageal speech influences the trans-source airflow rates, which in turn affects fundamental frequency and other acoustic variables.

The advantages of pulmonary air are reflected in an increased trans-source airflow rate, which helps reduce airway resistance (Moon \& Weinberg, 1987). These researchers found that these rates were variable among subjects with a range of $74-336 \mathrm{cc} / \mathrm{sec}$. Weinberg et al. (1982) found that TEP speakers had a trans-source airflow rate of 100-180 cc/sec during sustained productions of the vowel /a/. These values were considerably greater than those of conventional esophageal speakers who demonstrated a trans-source airflow rate of $27-72 \mathrm{cc} / \mathrm{sec}$ (Snidecor \& Isshiki, 1965).

When comparing TEP to laryngeal speakers, the rates of trans-source airflow were similar. Normal laryngeal speakers demonstrate trans-source airflow rates of 100-200 
cc/sec. Bless and Hirano (1982) found similar airflow rates for laryngeal speakers of 85-252 cc/sec.

\section{Airway Resistance}

Airway resistance cannot be precisely measured during any speaking method. To acquire this measurement, the pressure within the esophagus must be known. This cannot be done without positioning a sensing probe within the esophagus which requires the insertion of a probe through the voicing source. This is an invasive technique which is uncomfortable for the patient, has inherent physical risks, and may alter the voicing element (Weinberg et al., 1982). Tracheoesophageal speakers have demonstrated greater driving pressures and airway resistance compared to laryngeal speakers. Airway resistance measures for TEP speakers have been reported at $142-383 \mathrm{~cm} \mathrm{H} \mathrm{H}_{2} \mathrm{O} / \mathrm{LPS}$ (Moon \& Weinberg, 1987) and 155-270 $\mathrm{cm} \mathrm{H}_{2} \mathrm{O} / \mathrm{LPS}$ (Weinberg et al., 1982). These researchers also found source driving pressures to be 20-50 $\mathrm{cm} \mathrm{H}_{2} \mathrm{O}$ (Moon \& Weinberg, 1987). Weinberg et al. (1982) also found that a source driving pressure of 20-34 $\mathrm{cm} \mathrm{H}_{2} \mathrm{O}$ was needed to maintain voice for esophageal speakers. Laryngeal speakers, on the other hand, demonstrated airway resistance measures of $30-45 \mathrm{~cm}$ 
$\mathrm{H}_{2} \mathrm{O} /$ LPS and source driving pressures of $5-10 \mathrm{~cm} \mathrm{H}_{2} \mathrm{O}$ (Moon and Weinberg, 1987).

The reported measure of $20-35 \mathrm{~cm} \mathrm{H}_{2} \mathrm{O}$ of pressure is needed to sustain voice using traditional esophageal or TEP speech. Unfortunately, the mechanisms available to traditional esophageal speakers for generating and sustaining such pressures are limited. Active, exhalatory movements of the chest wall may increase driving pressures but may also increase the production of stoma noise through the open airway (Weinberg et al. 1982). Depletion of lung volume was also hypothesized to increase esophageal pressures. This may also increase the occurrence of stoma noise production which would negatively impact the production of words or syllable lengths per breath group during speech.

Moon and Weinberg (1987) found that all tracheoesophageal speakers in their study exhibited variations in fundamental frequency $\left(F_{0}\right)$ similar to laryngeal speakers. They argue that variability in $F_{0}$ cannot be predicted by the aerodynamic phenomenon only. They suggest that the myoelastic properties of the upper esophageal sphincter may also influence $F_{0}$ variations. Robbins, Fisher, Blom, and Singer (1984a) believe that the great variability in 
intersubject $F_{0}$ is also due to differences in surgical variations among the subjects. Moon and Weinberg (1987) argue that using $F_{\circ}$ to describe TEP and esophageal speech may not be a very effective method. Instead, they suggest it is the high degree of intersubject variability in average $F_{\circ}$ that describes the groups. Shipp (1967) states that higher measures of acceptability for esophageal speakers are related to a higher mean fundamental frequency, an increased rate, a greater proportion of periodic phonation, and a decreased proportion of both aperiodic phonation and silence. These variables could be measured and compared with perceptual judgements of speech acceptability.

The positive effects of an increased respiratory supply, a closed airway, and chest wall and background forces working together during TEP speech is apparent from this research. The individual using TEP speech is able to produce speech which has increased trans-source airflow rates, increased driving pressures, and decreased airway resistance. These characteristics have a direct effect on acoustic measures such as fundamental frequency, harmonicto-noise ratios and jitter and shimmer measurements. The 
impact of these measures on how people perceive the vocal quality of TEP speech has not been investigated.

\section{ACOUSTIC MEASURES}

Moon and Weinberg (1987) and Weinberg et al. (1982) suggest that the aerodynamic and myoelastic properties of alaryngeal and laryngeal speech influence acoustic speech signals. Because of this influence, an acoustic description of the physical qualities that create voice should be investigated to discover their effect on listener acceptance of alaryngeal speech methods.

\section{Fundamental Frequency}

Robbins (1984) devised a study to determine if speaker groups could be described by their vocal characteristics. This study was designed to investigate whether different acoustic variables could be used to effectively discriminate between esophageal, tracheoesophageal, and laryngeal speakers using a multivariate classifier. Seven intensity, 10 frequency, and 13 duration measures were quantified from recorded voice samples. Acoustical variables included mean $F_{\circ}$ during reading, $F_{0}$ range during /a/, jitter ratio, 
directional jitter (\%), mean shimmer, and directional shimmer $\left(\frac{\circ}{0}\right)$. The results indicated that speaker groups could be correctly identified with $100 \%$ accuracy by examining their acoustic and temporal patterns. The acoustic and temporal characteristic which most distinctly differentiated TEP speakers were increased intensity, reduced duration of phonation, and reduced rate. No significant differences in this study when comparing fundamental frequency $(102.8 \mathrm{~Hz}, 101.7 \mathrm{~Hz}$ respectively), directional jitter, mean shimmer, or words per phrase. Esophageal productions were differentiated from the other two comparison groups by $F_{0}$, jitter ratio, mean shimmer, maximum phonation time, and words per phrase. The acoustic and temporal similarities found between laryngeal and TEP speech again supports the role that the pulmonary air supply has for optimizing TEP speech when compared to esophageal speech.

Moon and Weinberg (1987) found large intersubject variability in fundamental frequency $\left(F_{0}\right)$ which ranged from 33 to $121 \mathrm{~Hz}$. Robbins, Fisher, Blom, and Singer (1984a) believe that the great variability in intersubject $E_{0}$ is also due to differences in surgical variations among the subjects. Moon and Weinberg (1987) argue that using 
Fo to describe TEP and esophageal speech acoustically may not be a very effective method. Instead, they suggest it is the high degree of intersubject variability in average $E_{0}$ that describes the groups. The effect of $F_{0}$ variatons on perceptual judgments of acceptability are unknown. This variable could be measured and compared with perceptual judgements of speech acceptability.

Although it has been found that TEP speech acoustically rated more similar to laryngeal speech in $F_{0}$ and intensity, naive listeners judged TEP speakers and esophageal speakers as not significantly different for pitch, quality, and loudness (Williams \& Watson, 1985). However, informed and expert listeners in the same study reported TEP speakers to be significantly superior in these areas. This suggests that the benefits of TEP speech aerodynamically and acoustically are not perceived auditorally by the average citizen. The average citizen is not trained to listen to very specific aspects of voice. They use their own individual guidelines which are created by life experiences and their own sense of vocal pleasantness. It is only by using a sample of untrained judges that we may understand how aerodynamic and acoustic 
properties play a role in the perception of alaryngeal speech.

\section{Jitter Measurements}

Very little research has been completed in the area of TEP speech and perturbation. Robbins, Fisher, Blom, and Singer (1984b) conducted a study of acoustic measures which included measures of perturbation. They found that TEP speakers produced a jitter ratio of 51.4. The TEP group's jitter ratio was considerably higher than the laryngeal group's measurement of 7.7 . These results are supported by the study done by Robbins (1984). Trudeau and Qi (1990) also had jitter ratio results that were higher for their female TEP group. They suggest that time postlaryngectomy and extent of surgery as possible variables which may increase perturbation ratios.

Although frequency perturbation measurements were considerably higher for TEP and esophageal speakers when compared to laryngeal speakers, the esophageal speakers demonstrated the highest values. Robbins (1984) and Robbins et al. (1984b) found esophageal measures to be 182.5 jitter ratio. Robbins et al. (1984b) also found mean jitter for esophageal speakers to be $4.1 \mathrm{~Hz}$. Smith, 
Weinberg, Feth, and Horii (1978) found mean jitter to range from .62 to $5.13 \mathrm{~Hz}$ and the jitter ratio to range from 39.53 to 48.88 . It is suggested by Smith, Weinberg, Feth, and Horii (1978) that the large magnitudinal differences produced by the esophageal group may suggest that the esophageal group employs a substantially different mechanism to regulate $F_{0}$ than TEP or laryngeal speakers. This theory is supported by Robbins et al. (1984b) who add that the mechanism employed by the esophageal group to regulate $F_{0}$ has less stability in $F_{0}$ control during sustained voicing whereas the TEP mechanism produces more variability during intentional $F_{0}$ changes of connected speech patterns.

\section{Shimmer Measurements}

Research in the area of shimmer has revealed that TEP speakers are similar to laryngeal speakers. Robbins (1984) and Robbins et al. (1984b) found mean shimmer to be .3 for TEP speakers. Horii (1980) found mean shimmer measurements for male laryngeal speakers to be .47 with a standard deviation of .34. Mean shimmer for normal female speakers was found to be .33 by Sorenson and Horii (1983). The female TEP group analyzed in the Trudeau and Qi (1990) 
study produced 1.9 mean shimmer. These results suggest that the magnitude of cycle-to-cycle amplitude variations for TEP speakers are similar to those of laryngeal speakers.

The differences in amplitude stability reflect the difference in the voicing mechanism as well as the physiological aspects of TEP and esophageal speech (Robbins et al., 1984). It is also suggested by these researchers that the variation in directional shimmer scores may reflect difficulty establishing a consistent interaction between the extremely high trans-source pressures and flows due to the limited control of the pharyngoesophageal segment.

Although Robbins (1984) found evidence that measures of fundamental frequency, intensity, and duration can be used to correctly identify alaryngeal speech type, there are no studies that claim that perturbation measures influence perceptual acceptance or identification of groups. Hoops and Noll (1969) completed a study with esophageal speakers and found that there was no connection between speech proficiency and frequency cycle-to-cycle variations (jitter). Wendahl (1966) found that increased jitter corresponded to perceptions of increased roughness 
in esophageal speech by judges. He also found that shimmer sounded more "bassy" but could be scaled for roughness as was jitter (Wendahl, 1966). His study used synthetic productions and is not comparable to the use of human voices. The use of synthetic voice allows for too much control over variables which are not controllable in human tissue.

Nine alaryngeal human subjects who used traditional esophageal speech participated in a study by Smith, Weinberg, Feth, and Horii (1978). This study investigated perturbation measures during prolonged /a/ productions. It found significantly more vocal jitter in the esophageal speech productions than in the laryngeal speech productions of sustained vowels and that listeners could reliably rate the severity of vocal roughness in the vowels. The perturbation measures, however, did not predict the perceived severity of vocal roughness by listeners. Studies evaluating shimmer and perceptual judgments for voices of human subjects were not found by this researcher.

In laryngeal voices, perturbation measures are used as an index of vocal stability and are a good tool for indentifying vocal pathologies. The cycle-to-cycle 
consistency of laryngeal vibration in phonation does not occur in a regular fashion (Laver, Hiller, \& Beck, 1992; Orlikoff \& Baken, 1993). Random variations (jitter and shimmer) of the cycle-to-cycle period in the normal voice are small. Larger variability in the cycle-to-cycle periods increase perturbation measures and contribute to the listener's perception of vocal roughness or harshness. There are no studies comparing certain acoustic measures to perceptual judgements of speech acceptability for tracheoesophageal vocal productions. This research will attempt to determine whether a relationship does exist. If a correlation between perturbation measures and perceptual judgments is found, it would imply that jitter and shimmer measures influence listeners' perceptions of voice quality produced by TEP speakers.

Jitter and shimmer measures selected for this study are related to perceptions of harsh, hoarse, or rough voice quality and have been used in voice evaluations to determine vocal pathologies(Orlikoff \& Baken, 1993). Judgments of vocal roughness may influence the choice of communication selected by laryngectomees. It could also provide insight for treatment techniques and strategies. 
These are important areas to the patient who is trying to improve communication interaction.

\section{PERCEPTUAL JUDGMENTS}

Aerodynamic and acoustic findings suggest that TEP speech is superior to esophageal speech. It has also been described as more similar to laryngeal speech than esophageal. Shipp (1967) suggests that alaryngeal speech which has a higher $F_{0}$, less respiratory noise, and less silence within an utterance with higher phonation time would be rated as more acceptable. These qualities are all described in TEP measurements. Perceptual ratings suggest that TEP, with all its advantages, is not always rated as more acceptable. Variations in study design may have influenced these apparently contradictory results.

Although TEP speech has been measured to have less perturbation than esophageal speech, it is not rated in studies as more acceptable. Trudeau (1987) did a comparison of speech acceptability between good and excellent TEP and esophageal speakers. His results show that TEP speakers were not rated as more or less acceptable in speech than esophageal speakers. He suggests that the acoustic and temporal dissimilarities 